\title{
RADIATION DAMAGE IN POLARIZED TARGET MATERIALS*
}

\author{
RICHARD C. FERNOW \\ Randall Laboratory of Physics, University of Michigan, Ann Arbor, Michigan 48109, U.S.A.
}

Received 2 August 1977

We examine some of the parameters which influence the decay of the polarization of polarized targets in an intense proton beam. We find that the usual characteristic flux for the polarization to drop to $1 / \mathrm{e}$ of its initial value does not provide a sufficient criterion to specify the radiation resistance of target materials. We give a more detailed method of evaluation including an empirical formula for the effect of annealing on the target polarization.

During the course of a recent high energy physics experient ${ }^{1}$ ) we have accumulated a significant amount of data on the radiation resistance of diol and butanol polarized targets. The experiment was performed in an external proton beam at the Z.G.S. at Argonne National Laboratory. The results allow a better comparison of the suitability of various target materials when the target must be subjected to a high intensity beam.

Diols have been used as polarized target materials because of their high proton polarization and relatively short polarizing times ${ }^{2}$ ). A diol is a hydrocarbon chain with two of the hydrogens replaced by $-\mathrm{OH}$ groups. The diols examined here are 1,2-ethanediol, $\mathrm{OHC} \mathrm{H}_{2} \mathrm{CH}_{2} \mathrm{OH}$, and 1,2-propanediol, $\mathrm{CH}_{3} \mathrm{CH} \mathrm{OHCH}_{2} \mathrm{OH}$. The target samples were prepared by mixing the diol and potassium dichromate under carefully controlled conditions of temperature, light, and pressure. The procedure was designed to give an optimum concentration of paramagnetic $\mathrm{CrV}$ complexes in the diol solution. Table l lists several properties of the diol samples: the liquid EPR derivative peak separation and the

\footnotetext{
* Work supported in part by the U.S. Energy Research and Development Administration.
}

corresponding concentrations, the annealing temperatures, the nuclear relaxation times at $0.5^{\circ} \mathrm{K}$ before and after irradiation, and the initial polarization values.

The target material was frozen into beads approximately $1.5 \mathrm{~mm}$ in diameter and placed in a $4.13 \mathrm{~cm}$ long by $2.89 \mathrm{~cm}$ diameter teflon holder. The bead filling fraction was 0.627. Inside the holder were two NMR coils for the target polarization measurement. The first or small coil was just a straight piece of $0.4 \mathrm{~mm}$ diameter wire with $2.5 \mathrm{~mm}$ o.d. teflon insulation. The second or large coil was a helix of the same wire $12 \mathrm{~mm}$ in diameter with $11 \mathrm{~mm}$ spacing between the loops. The coils were connected to separately tunable LRC series resonance circuits and were swept thru $385 \mathrm{kHz}$ around the central frequency of $106.8 \mathrm{MHz}$.

The target was placed in a $25.084 \mathrm{kG}$ magnetic field and maintained at $0.48 \mathrm{~K}$ with a ${ }^{3} \mathrm{He}$ evaporation cryostat. The polarization was dynamically enhanced at $69.30 \mathrm{GHz}$ and $69.62 \mathrm{GHz}$ using $\sim 15 \mathrm{~mW}$ of mizrowave radiation. The microwaves entered at the bottom of the $4.29 \mathrm{~cm}$ long, $3.09 \mathrm{~cm}$ i.d., and $0.13 \mathrm{~mm}$ thick copper cavity thru a RG98/U silver waveguide.

TABLE 1

Properties of the diol target materials.

\begin{tabular}{lcccccc}
\hline & $\begin{array}{c}\Delta H_{\mathrm{c}} \\
(\mathrm{G})\end{array}$ & $\begin{array}{c}\left.\text { Conc. }{ }^{2,15}\right) \\
\left(\times 10^{19} \mathrm{spins} / \mathrm{cm}^{3}\right)\end{array}$ & $\begin{array}{c}T_{\text {anneal }} \\
(\mathrm{K})\end{array}$ & $\begin{array}{c}T_{\ln }(0.5 \mathrm{~K}) \\
(\min )\end{array}$ & $\begin{array}{c}T_{\ln }^{\mathrm{rr}}(0.5 \mathrm{~K}) \\
(\mathrm{min})\end{array}$ & $\begin{array}{c}p_{0} \\
(\%)\end{array}$ \\
\hline Ethanediol & 5.2 & 8.0 & 160 & 277 & 220 & 81 \\
Propanediol & 5.4 & 11 & 180 & 164 & 761 \\
\hline
\end{tabular}


After irradiation the target polarization could be partially restored by annealing the material. The heat was provided by 19 turns of enamelled $4 \mathrm{mil}$ constantin wire wrapped around the microwave cavity. The heater supplied up to $35 \mathrm{~W}$ of power for rapid annealing. The diol samples discussed here were typically heated from $0.5 \mathrm{~K}$ to $160-180 \mathrm{~K}$ in $8-13 \mathrm{~min}$.

The incident proton flux on the target was monitored with a three counter scintillation telescope. The absolute normalization was provided by calibrating the counter telescope with flux measurements from aluminum foils exposed to the incident beam. The induced $\beta^{+}$activity from ${ }^{18} \mathrm{~F}$ produced in the foils was counted in a calibrated $\mathrm{NaI}$ well counter. The horizontal and vertical profiles of the beam incident on the target were measured with segmented wire ion chambers (SWIC) located just upstream of the polarized target.

The actual measurements for one ethanediol sample are shown in fig. 1. As mentioned before, these measurements were made during the course of a high energy physics experiment. One constraint was that the average polarization be kept as high as possible and this required that we anneal the target frequently and change the target material at least once per week. As a consequence most individual measurements of the slope had rather large errors. However, many measurements were made on each sample and the resultant weighted averages are significant.

The points plotted in fig. 1 indicate the average of the readings on the large and small coils. We have only used polarization values recorded after the target had reached its maximum polarization.

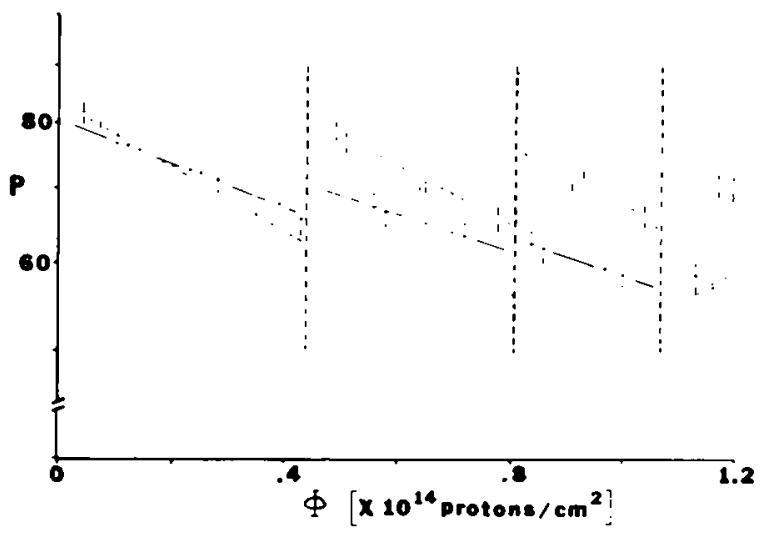

Fig. 1. The polarization of one of the ethanediol samples is shown as a function of the accumulated radiation flux. The two symbols represent positive and negative enhancements Dotted lines indicate places where the target was annealled.
The polarizations were corrected for baseline curvature and dispersion in the NMR signal. It was difficult to keep the NMR frequency, magnetic field, microwave frequency, and microwave power simultaneously and optimally in tune, especially since the optimal parameters seemed to vary with radiation damage $\left.{ }^{3}\right)$. The parameters were retuned after each target reversal and we estimate the polarization was within $2 \%$ of maximum for most measurements. This error only applies to the upper error on the polarization. We estimate an error of $\pm 0.2 \%$ on the dispersion correction, $\pm 0.5 \%$ on the baseline correction and $\pm 2 \%$ from beam movement. From these considerations we have assigned an error of $-\frac{3}{2} \%$ to all the polarization measurements.

The number of monitor counts gives the integrated intensity, $\varphi\left[\# / \mathrm{cm}^{2}\right]$, of the beam striking the target. However, the relevant quantity for the absisca in fig. 1 is the integrated beam flux, $\Phi\left[\because / \mathrm{cm}^{2}\right]$. We have empirically determined the flux distribution function to be

$\Phi(x, y)=\Phi_{0}\left(1-\frac{x^{2}}{A^{2}}-\frac{y^{2}}{B^{2}}\right)^{4}$,

where $\Phi_{0}$ is the flux on the beam axis, and $A$ and $B$ are the semi-widths of the elliptical beam cross
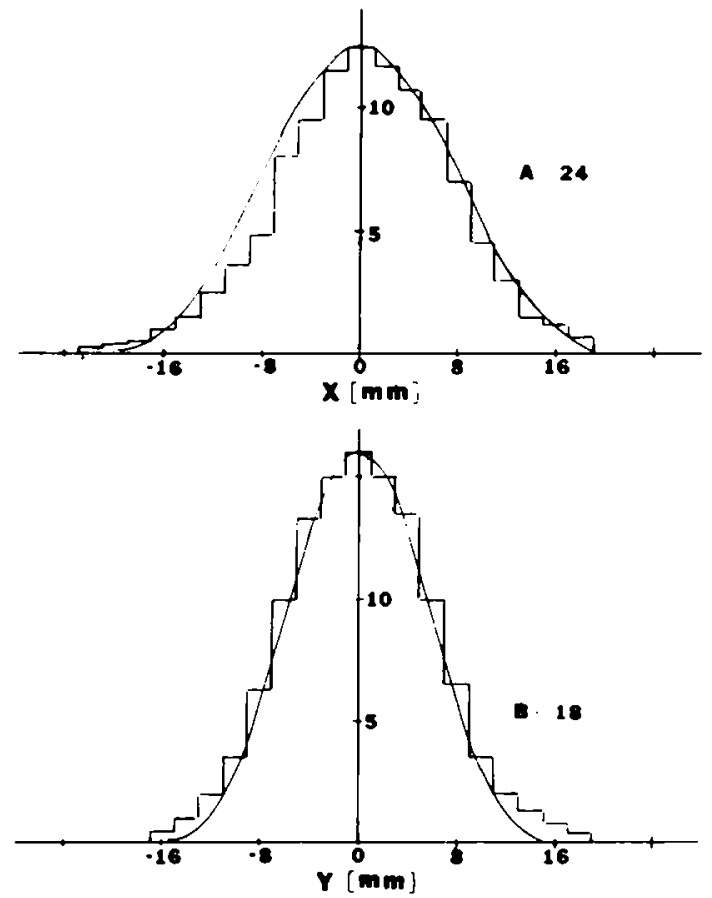

Fig. 2. The horizontal and vertical beam profiles at the polarized target. The curves indicate the integrals of eq. (1) with the given values of the parameters $A$ and $B$. 
section. This assumed distribution was checked by integrating eq. (1) and comparing the resultant function with the observed SWIC distributions. The agreement is satisfactory as shown in fig. 2. Integrating eq. (1) over the elliptical beam profile gives

$\Phi_{0}=\frac{5 \varphi}{\pi A B}$.

In principal we could use eq. (1) to compare the flux at each coil with the polarization measured there. Unfortunately the beam tended to jump around several $\mathrm{mm}$ on a pulse to pulse basis. This plus the inevitable problems of keeping the beam steered precisely on the target axis have prevented us from making two independent measurements. Instead we have averaged the polarization values and used the flux that is appropriate to a point midway between the coils.

We have assumed that the polarization decay followed the standard form

$p(\Phi)=p_{0} \mathrm{e}^{-\Phi / \Phi_{A}}$,

where $p(\Phi)$ is the polarization after being irradiated with $\Phi$ protons $/ \mathrm{cm}^{2}, p_{0}$ is the starting polarization, and $\Phi_{\mathrm{A}}$ is the characteristic flux ${ }^{4}$ ) for the polarization to decrease by $1 / \mathrm{e}$. One iso-butanol sample was irradiated for $5 \times 10^{14}$ protons $/ \mathrm{cm}^{2}$ before annealing. The sample appears to follow one exponential for the first $10^{14}$ protons $/ \mathrm{cm}^{2}$ and then a second less steep exponential for the next $4 \times 10^{14}$ protons $/ \mathrm{cm}^{2}$. Since in practice one would not run more than $10^{14}$ protons $/ \mathrm{cm}^{2}$ into a target without annealing eq.(3) gives a good representation of the data.

We checked to see if there was any evidence that $\Phi_{\mathrm{A}}$ was a function of the accumulated flux but found that within the errors $\Phi_{\mathrm{A}}$ could be con- sidered a constant for accumulated fluxes less than $2 \times 10^{14}$ protons $/ \mathrm{cm}^{2}$. We were also unable to find any dependence of $\Phi_{\mathrm{A}}$ on the instantaneous flux for fluxes $(1.0-3.0) \times 10^{9}$ protons $/ \mathrm{cm}^{2}$. pulse.

The dotted lines in fig. 1 indicate points where the target was annealed. Qualitatively it can be seen that although the material always responded to the annealing process, it became less effective after a period of time. We present average values for the diol characteristic fluxes in table 2. The quoted errors do not include the estimated $8 \%$ uncertainty in the absolute normalization of the proton intensity measurements or $5 \%$ uncertainty in the effective beam cross. section.

It is clear from table 2 that the two enhancements damage at different rates ${ }^{5}$ ). For both diols the positive enhancement appears to suffer less anneallable radiation damage than the negative enhancement. In addition the ethanediol samples measured here appear to have significantly better radiation resistance than the propanediol. An earlier measurement ${ }^{6}$ ) of $\Phi_{\mathrm{A}}$ for ethanediol at $1 \mathrm{~K}$ is also shown in table 2. Our results for 1,2-propanediol are lower than earlier measurements ${ }^{7}$ ) at $\frac{1}{2} \mathrm{~K}$. This could be the result of differing concentrations and points out that to be useful radiation damage measurements should give some indication of the concentration of the irradiated material.

The fact that the polarizations shown in fig. 1 do not return to their initial values after several annealing periods shows that more than one type of damage mechanism is present. This can be seen more clearly in fig. 3 where we show the extrapolated polarization values immediately following annealing as a function of $\Phi$ for the negative enhancement data from ethanediol. The polarization following annealing steadily declines for some

TABLE 2

Radiation damage summary for diol targets.

\begin{tabular}{|c|c|c|c|c|c|c|}
\hline Material & $\begin{array}{c}\Phi_{A}^{+} \\
\left(\times 10^{14} \mathrm{p} / \mathrm{cm}^{2}\right)\end{array}$ & $\left(\times 10^{14} \mathrm{p} / \mathrm{cm}^{2}\right)$ & $\begin{array}{c}\Phi_{N_{A}}^{+} \\
\left(\times 10^{14} \mathrm{p} / \mathrm{cm}^{2}\right)\end{array}$ & $\begin{array}{c}\Phi_{\overline{\overline{\mathrm{N}}_{\mathrm{A}}}} \\
\left(\times 10^{14} \mathrm{p} / \mathrm{cm}^{2}\right)\end{array}$ & $p_{\mathrm{a}}^{+} / p_{0}^{+}$ & $p_{\mathrm{a}}^{-/} p_{0}$ \\
\hline Ethanediol & $\begin{array}{r}2.33^{+0.32}-0.17\end{array}$ & $\begin{array}{r}1.75+0.18 \\
-0.13\end{array}$ & $0.76 \pm 0.16$ & $0.80 \pm 0.15$ & $0.69 \pm 0.03$ & $0.89 \pm 0.02$ \\
\hline Propanediol & $\begin{array}{r}1.24+0.43 \\
-0.12\end{array}$ & $0.94^{+0.12}-0.07$ & $0.80 \pm 0.25$ & $1.00 \pm 0.30$ & $0.73 \pm 0.04$ & $0.90 \pm 0.04$ \\
\hline $\begin{array}{l}\left.\text { Ethanediol }{ }^{6}\right) \\
{\left[+3 \% \mathrm{H}_{2} \mathrm{O}\right]}\end{array}$ & $1.64 \pm 0.10$ & $1.82 \pm 0.17$ & & & & \\
\hline Propanediol ${ }^{7}$ ) & $2.0 \pm 0.25$ & $1.6 \pm 0.20$ & & & & \\
\hline
\end{tabular}


characteristic amount of flux and then appears to be unaffected by further irradiation. We have parameterized this behavior with the empirical formula

$p(\Phi)=p_{\mathrm{a}}+\left(p_{0}-p_{\mathrm{a}}\right) \mathrm{e}^{-\Phi / \Phi_{N A}}$,

where $p_{\mathrm{a}}$ is the "asymptotic" polarization for large $\Phi, p_{0}$ is the initial polarization, and $\Phi_{\mathrm{NA}}$ is the characteristic flux for this non-anneallable damage to reach $1 / \mathrm{e}$ of its asymptotic value. We consider the ratio $p_{\mathrm{a}} / p_{0}$ and the characteristic flux $\Phi_{\mathrm{NA}}$ to be useful figures of merit when comparing the radiation resistance of target materials and show the results for both diols and both enhancements in table 2. The fluxes $\Phi_{\mathrm{NA}}$ appear to be similar in magnitude to the $\Phi_{\mathrm{A}}$ values. Also the ratio of asymptotic to initial polarizations appears to be about $70 \%$ for positive enhancement and $90 \%$ for negative enhancement for both materials.

In an effort to find a more resistant target material we have followed a suggestion by $\mathrm{Ash}^{8}$ ) and examined the isomers of butanol. The results shown in table 3 indicate that the radiation resistance for dynamic polarization is at least qualitatively proportional to the resistance to form color centers $^{9}$ ). Unfortunately there may also be an inverse relation between radiation resistance and the magnitude of the polarization. The data for isobutanol also indicates that the addition of water to the sample to increase the solubility of the porphyrexide may adversely affect the radiation resistance of the sample.

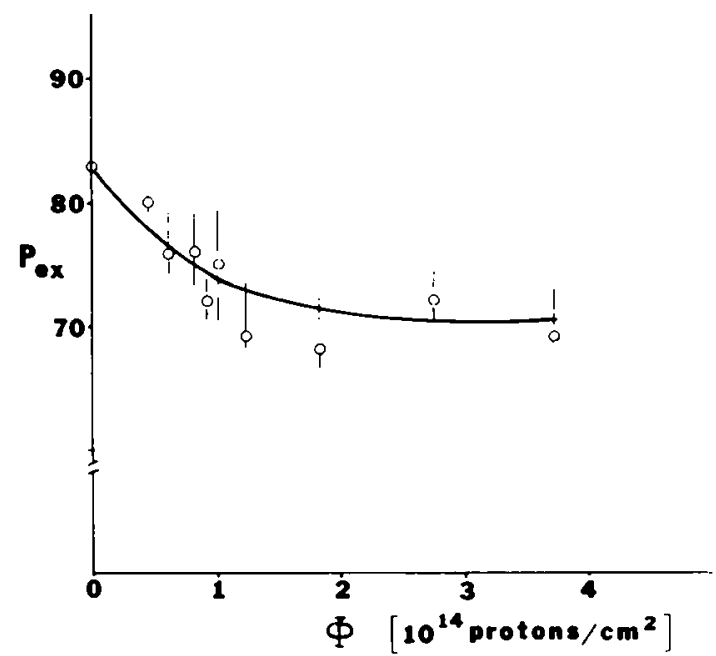

Fig. 3. The extrapolated polarization immediately following annealling is shown as a function of the accumulated radiation flux for the negative enhancement data for ethanediol. The curve is eq. (4) with the parameters given in table 2.
TABLE 3

Butanol radiation damage summary: $\mathrm{PX}=$ porphyrexide.

\begin{tabular}{|c|c|c|c|c|c|}
\hline Material & Doped & $p_{\max }^{+}$ & $p_{\max }^{-}$ & $\Phi_{A}^{+}$ & $\Phi_{\bar{A}}^{-}$ \\
\hline n-butanol & $\begin{array}{l}5 \% \mathrm{H}_{2} \mathrm{O} \\
1 \% \text { PX }\end{array}$ & 68 & -62 & $2.2 \pm 0.5$ & $4.5 \pm 1.0$ \\
\hline \multirow[t]{3}{*}{ iso-butanol } & $\begin{array}{l}2 \% \mathrm{H}_{2} \mathrm{O} \\
1 \% \mathrm{PX}\end{array}$ & 51 & & & \\
\hline & $\begin{array}{l}5 \% \mathrm{H}_{2} \mathrm{O} \\
1 \% \mathrm{PX}\end{array}$ & 65 & -58 & $3.2 \pm 1.0$ & $6.8 \pm 3.0$ \\
\hline & $\begin{array}{l}8 \% \mathrm{H}_{2} \mathrm{O} \\
1.5 \% \mathrm{PX}\end{array}$ & 45 & -55 & $1.5 \pm 0.4$ & $2.5=0.6$ \\
\hline \multicolumn{6}{|l|}{ sec-butanol } \\
\hline & $\begin{array}{l}5 \% \mathrm{H}_{2} \mathrm{O} \\
1 \% \mathrm{PX}\end{array}$ & 62 & -51 & $2.7 \pm 0.3$ & $6.1=1.5$ \\
\hline tert-butanol & $\begin{array}{l}5 \% \mathrm{H}_{2} \mathrm{O} \\
1 \% \mathrm{PX}\end{array}$ & $\sim 10$ & & & \\
\hline
\end{tabular}

It may be worthwhile to briefly review the currently accepted ideas on the mechanism of radiation damage. Irradiation of amorphous solids generally results in the partial dissociation of the solid matrix and production of trapped electrons and free radicals. Five to six paramagnetic particles are created in ethanediol for each $100 \mathrm{eV}$ of absorbed dose ${ }^{10}$ ). Electrons may be trapped in inter-molecular cavities formed by groups of favorably oriented molecules. However the amplitude of the EPR line for these trapped electrons saturates and decreases at very small microwave powers ${ }^{11}$ ).

The more serious problem appears to be radical formation. Radical concentrations typically reach $10^{18}-10^{20}$ radicals $/ \mathrm{cm}^{3}$ in organic solids ${ }^{12}$ ). In the diols radicals are formed mainly by the elimination of an $\alpha$ hydrogen atom. For example $\mathrm{HOCH}_{2} \mathrm{CHOH}$ is known to be created in ethanediol and to interact via the hyperfine interaction with undamaged hydrogen atoms ${ }^{13}$ ).

Annealling is effective at depleting the trapped electron and radical concentrations. The heat provides increased molecular mobility leading to ionic recombination and reactions with molecules. In the viscous substances discussed here radical concentrations are reduced to less than $1 \%$ of the original concentration by heating to $0.6-0.7$ of the melting temperature ${ }^{14}$ ). Trapped electrons are released at lower temperatures than radicals.

We have looked for some evidence of dynamic nuclear polarization from the produced radicals. Fig. 4 shows the dependence of the nuclear polarization on the microwave frequency for (1) a nonirradiated ethanediol sample, (2) an irradiated sam- 


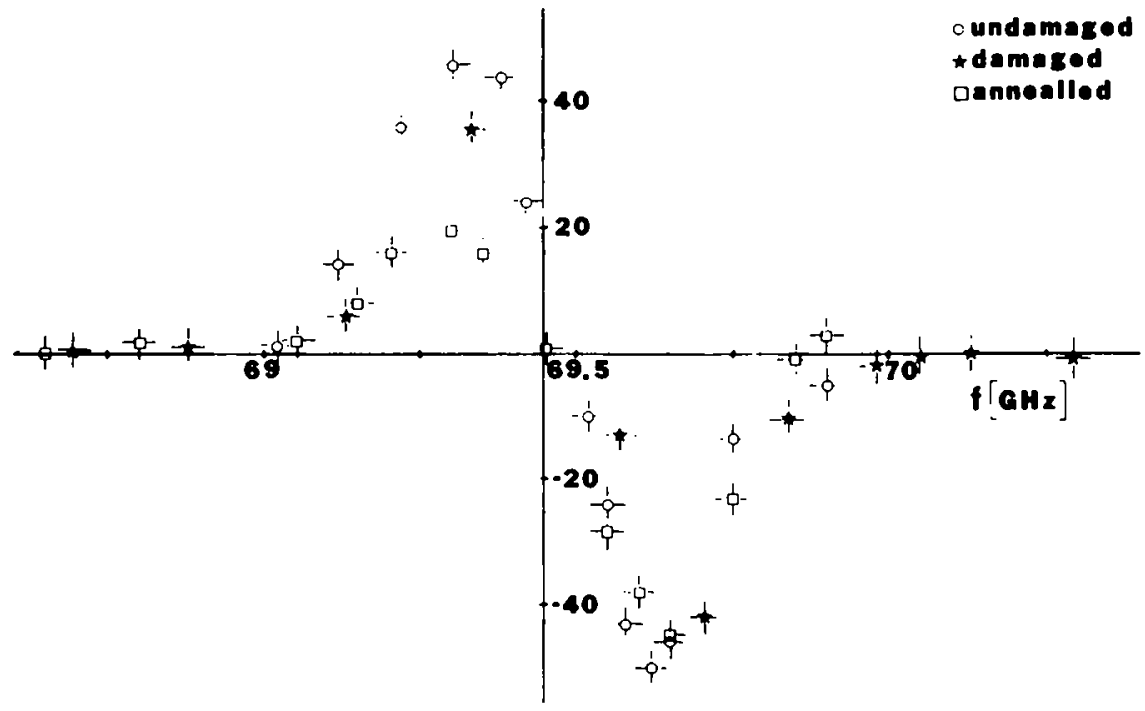

Fig. 4. The proton polarization at $0.8^{\circ} \mathrm{K}$ is shown as a function of the microwave frequency for a normal ethanediol sample, a damaged sample, and a damaged but annealed sample. The peaks of the damaged curve were maximized before the frequency measurements. The absolute magnitude of the normal and annealed samples are arbitrary.

ple, and (3) an irradiated sample that was annealled to $143 \mathrm{~K}$. Within the uncertainty in the frequency measurement the positive enhancement (low frequency) polarization peaked in the same place for all three samples. The negative enhancement polarization for the annealled sample peaked one standard deviation higher than the normal sample, while the damaged sample peaked three standard deviations higher. However there was no evidence of any induced polarization greater than $2 \%$ in the tails of the diol signal and in particular near $70.17 \mathrm{GHz}$ which corresponds to $g=2$.

The shifting of the diol negative enhancement peak and the values for $\Phi_{\mathrm{A}}$ in tables 2 and 3 suggest the following qualitative scheme for the effect of radiation on dynamic polarization. First the radiation may reduce the polarization of both enhancements roughly equally by creating molecular fragments, liberated hydrogen, etc. which can interact with the proton spin system and short-circuit the dynamic electron-proton spin-spin coupling. This could cause the observed shorter relaxation times in the damaged samples. Secondly, free radicals and trapped electrons (which are produced with $g \approx 2$ ) could modify and broaden the EPR spectrum of the CrV. These new paramagnetic entities would have the greatest effect on the negative enhancement of the diols and positive enhancement of the forms of butanol. Examination of tables 2 and 3 shows that $\Phi_{\mathrm{A}}^{-}<\Phi_{\mathrm{A}}^{+}$for the diols and $\Phi_{\mathrm{A}}^{+}<\Phi_{\mathrm{A}}^{-}$for all the isomers of butanol. In addition as mentioned above the radicals can be largely removed by annealling.

I would like to thank the authors of ref. 1 and J. Bywater, J. Norris, D. Hill and A. Moretti of Argonne National Laboratory for their assistance during the acquisition of the data presented in this paper.

\section{References}

l) H. Miettinen, K. Abe, R. Fernow, A. Krisch, T. Mulera, A. Salthouse, B. Sandler, K. Terwilliger, J. O'Fallon, L. Ratner and P. Schultz, Phys. Rev. D16 (1977) 549

2) W. de Boer, Nucl. Instr. and Meth. 107 (1973) 99; CERN Report 74-11 (1974).

3) The microwave frequency for maximum polarization in the negative enhancement appeared to shift to higher frequencies as the samples became damaged. There was no apparent shift in the positive enhancement frequency.

4) We use the subscript A to indicate that on a short term basis most of this damage could be annealled away.

5) We define positive enhancement to be in the same direction as the thermal equilibrium signal.

6) H. Petri and G. Abshire, Nucl. Instr. and Meth. 119 (1974) 205. The authors prepared their ethanediol samples with $3 \%$ water and $2 \%$ potassium dichromate whereas we prepared ours with no water and $7.9 \%$ potassium dichromate. Hence it would appear that our sample was more concentrated.

7) D. Crabb, in High energy physics with polarized beams and targets (ed. M. Marshak; AIP Conf. Proc. No. 35, 1976) p. 120. 
8) W. Ash, in ref. 7, p. 485.

$\left.{ }^{9}\right)$ R. Alger et al., J. Chem. Phys. 30 (1959) 695.

10) S. Pshezhetskii et al., EPR of free radicals in radiation chemistry (J. Wiley, New York, 1974) p. 198

11) S. Pshezhetskii et al., op. cit, p. 84.
12) S. Pshezhetskii et al., op. cit, p. 77.

13) S. Pshezhetskii et al., op. cit, p. 195.

14) S. Pshezhetskii et al., op. cit, p. 301.

15) H. Glätti, Proc. 2nd Int. Conf. on Polarized targets. (1971) p. 281. 